\title{
FIB-TOF Tomography of Solid Oxide Fuel Cells
}

\author{
John S. Hammond ${ }^{1}$, Gregory L. Fisher ${ }^{1}$, Scott R. Bryan ${ }^{1}$, Rait Kanarbik² and Pritt Möller ${ }^{2}$ \\ 1. Physical Electronics, 18725 Lake Drive East, Chanhassen, MN 55317 \\ 2. Institute of Chemistry, University of Tartu, 50090 Tartu, Estonia
}

Time-of-flight secondary ion mass spectrometry (TOF-SIMS) is a surface-sensitive chemical imaging technique in which the outermost $\sim 2 \mathrm{~nm}$ of the surface is sampled. Characterization of materials in the range of several microns from the sample surface has become somewhat routine with the use of a sputter ion beam to remove multiple layers of material between analysis (chemical imaging) cycles. Nevertheless, there are practical limitations to the use of ion beam sputtering for probing inorganic specimens beyond the surface region. Certain matrix components do not sputter well and are susceptible to ion beam-induced chemical damage. This accumulated beam damage gives rise to incorrect chemical distributions. Some matrix components may sputter at a different rate than others which results in a misrepresentation of the chemical distributions. Utilizing extremely low energy sputter ion beam energies in order to minimize the aforementioned artifacts, the analytical time requirements can become prohibitive. Finally, the utility of sputter depth profiling for 3D TOF-SIMS imaging is limited to a few microns in the case of a favorable sample matrix and to a couple hundred nanometers in the case of an unfavorable sample matrix.

An alternative approach to achieve 3D chemical imaging is to utilize in situ FIB milling and sectioning in conjunction with TOF-SIMS chemical imaging... what we have called FIB-TOF tomography. With FIB milling, the interior of a specimen is revealed to depths of $\geq 50 \mu \mathrm{m}$. Additionally, 3D chemical imaging of $>10 \mu \mathrm{m}$ deep volumes may be achieved within reasonable analytical times. The advantage of the in situ FIB-TOF approach is that the artifacts caused by sputter depth profiling such as differential sputtering and accumulated ion beam mixing are avoided. The union of successive FIB sectioning and TOF-SIMS analysis cycles, with the sample maintained in one analytical position to achieve 3D chemical imaging, will be discussed for the analysis of solid oxide fuel cell (SOFC) samples.

Figure 1a and $1 \mathrm{~b}$ shows the secondary electron images produced by the analytical LMIG imaging of the face of the initial FIB cut. The highly porous cathode and first electrolyte layers would produce severe artifacts using traditional 3D TOF-SIMS imaging. The FIB-TOF approach also allows the cathode, two electrolyte layers and the anode to be imaged within a depth of $\geq 50 \mu \mathrm{m}$ from the sample surface.

Figure $2 \mathrm{a}$ and $2 \mathrm{~b}$ show element specific $3 \mathrm{D}$ images of the cathode and two electrolyte layers using the FIB-TOF technique. The presence of $\mathrm{K}$ decorating the void structures, the heterogeneous composition of the first electrolyte layer and the segregation of Sr between the first and second electrolyte layer can be easily observed.

The presentation will explain the FIB-TOF instrumentation based on a FIB LMIG, an imaging analytical LMIG and the TRIFT TOF mass spectrometer. The presentation with also provide further insights on the 3D compositional analysis afforded by the high mass resolution, high mass accuracy and high spatial resolution data provided by the FIB-TOF technique. 

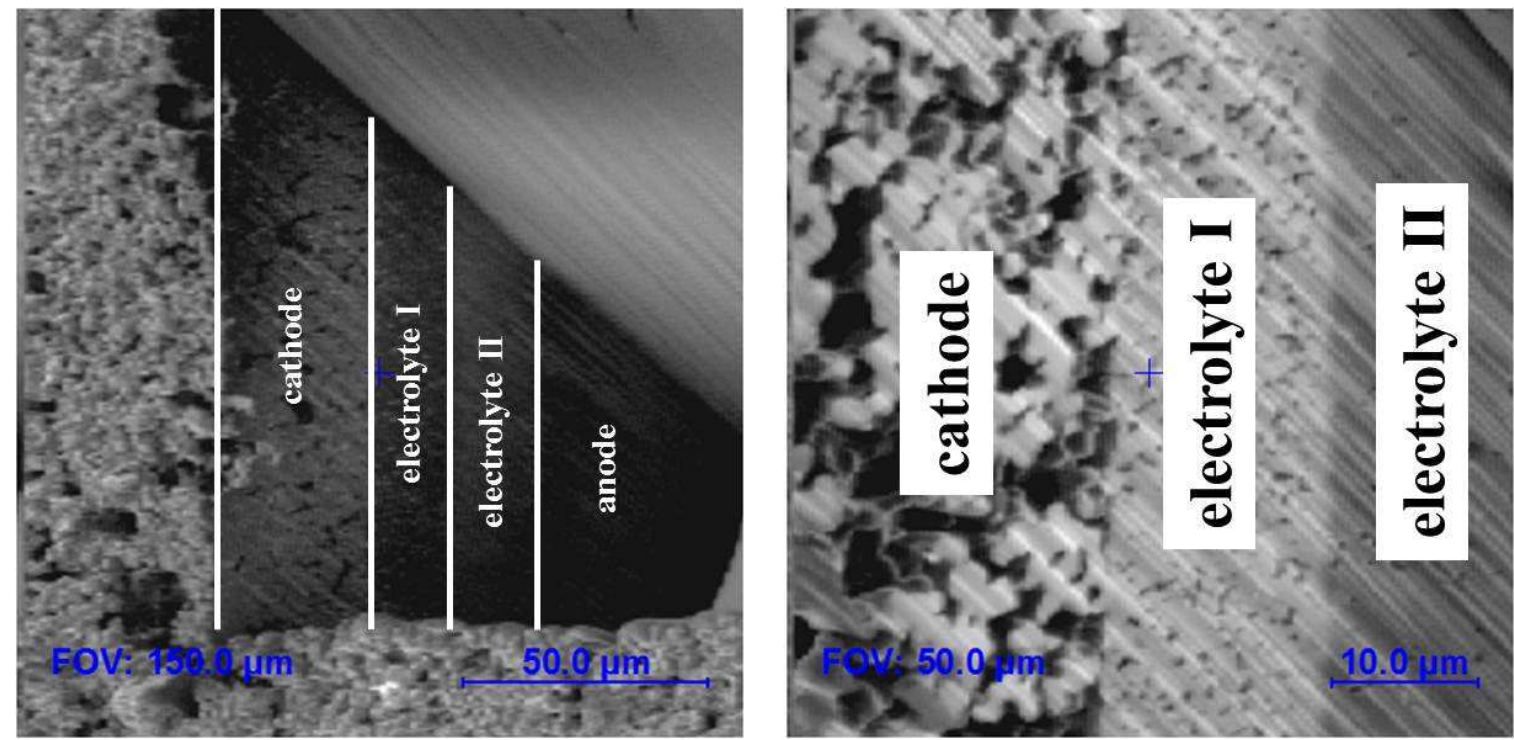

Figure 1a (left) and Figure 1b (right) show $150 \mu \mathrm{m}$ and $50 \mu \mathrm{m}$ FOV secondary electron images respectively induced by the analytical LMIG following the initial FIB cut into the SOFC.
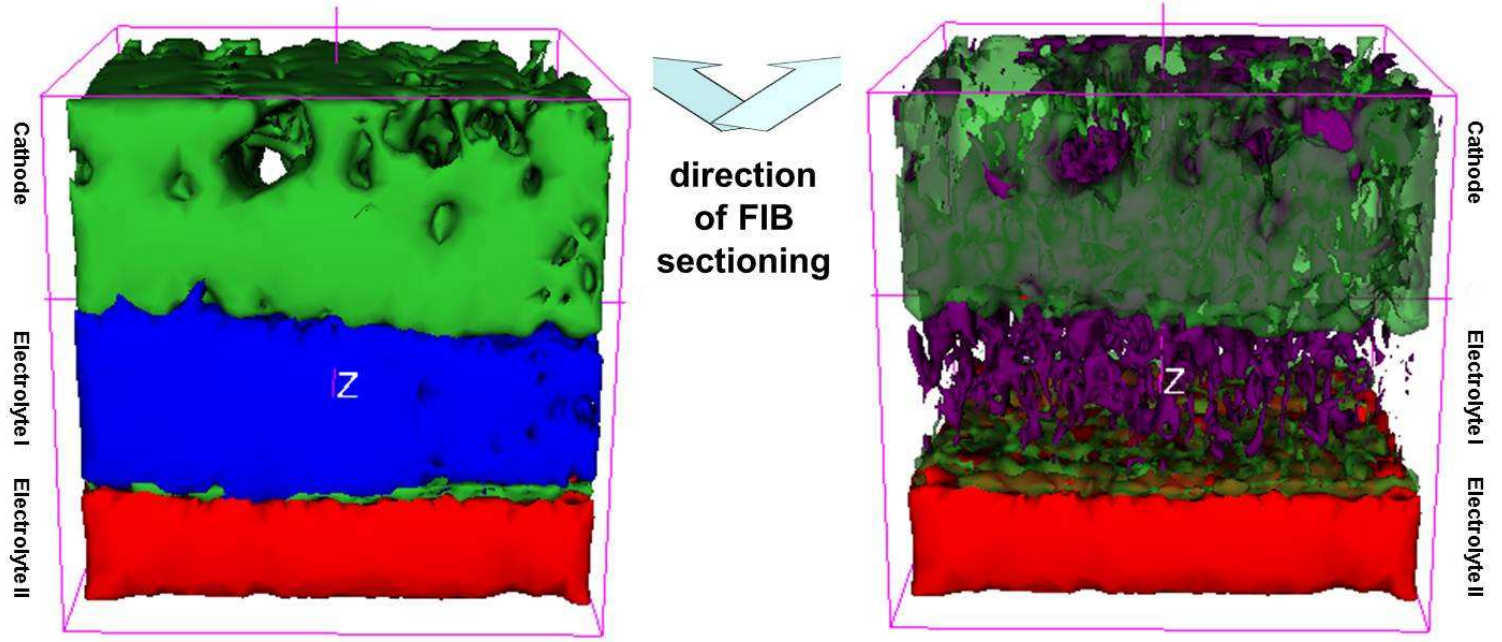

Figure 2a (left) shows the 3D TOF-SIMS images of $\mathrm{Sr}^{+}, \mathbf{C e}^{+}$, and $\mathrm{Zr}^{+}$. Figure $\mathbf{2 b}$ (right) shows the 3D TOF-SIMS images of $\mathrm{Sr}^{+}, \mathrm{K}^{+}$, and $\mathrm{Zr}^{+}$. Of particular note is the presence of high levels of $\mathrm{K}$ decorating the voids of the cathode as well as the heterogeneous distribution of $\mathrm{K}$ in the first electrolyte layer. 